\title{
Escherichia coli tryptophan repressor binds multiple sites within the aroH and trp operators
}

\author{
Andrew A. Kumamoto, ${ }^{1}$ William G. Miller, ${ }^{2}$ and Robert P. Gunsalus ${ }^{1,2}$ \\ ${ }^{1}$ Molecular Biology Institute and the ${ }^{2}$ Department of Microbiology, University of California, Los Angeles, California 90024 \\ USA
}

\begin{abstract}
DNase I footprinting and methylation protection studies have been used to analyze the binding of Escherichia coli Trp repressor to the $\operatorname{trpR}$, aroH, and trp operators. The methylation protection assay shows that Trp repressor binds in two successive major grooves of the $\operatorname{trp} R$ operator, three successive major grooves of the aroH operator, and four successive major grooves of the trp operator. The simplest model that explains the difference in Trp repressor interaction at the three operators is that the aroH and trp operators are composed of multiple, helically stacked binding sites. When viewed in three dimensions, each site is positioned on a different face of the DNA, and together process up the surface of the DNA helix. Analysis of a deletion derivative of the trp operator supports this model.
\end{abstract}

[Key Words: Trp repressor; aroH operator; trp operator; repressor binding]

Received February 23, 1987; revised version accepted June 6, 1987.

The Trp repressor of Escherichia coli coordinately regulates the expression of the trp, $a r o H$, and $\operatorname{trpR}$ operons in response to the intracellular levels of L-tryptophan (Cohen and Jacob 1959; Brown 1968; Rose et al. 1973; Gunsalus and Yanofsky 1980). The trpR operon, located at $99.5 \mathrm{~min}$, codes for the Trp aporepressor and is autoregulated (Morse and Yanofsky 1969; Gunsalus and Yanofsky 1980; Kelley and Yanofsky 1982; Bachman 1983; Gunsalus et al. 1986). The aroH operon, located at 37 min, encodes one of the three isozymes of 3-deoxyD-arabino-heptulosonic acid-7-phosphate synthetase (DAHP). This enzyme catalyzes the first step in the common pathway of aromatic amino acid biosynthesis (Brown 1968; Pittard et al. 1969; Zurawski et al. 1981). The third operon, trp, is located at $27 \mathrm{~min}$ on the E. coli genetic map and encodes the enzymes that carry out the final steps of L-tryptophan biosynthesis from the cellular intermediate, chorismic acid (Yanofsky 1971).

The native form of the Trp aporepressor is a dimer composed of identical subunits of 108 amino acids (Gunsalus and Yanofsky 1980; Joachimiak et al. 1983; Arvidson et al. 1986). Binding of the corepressor, L-tryptophan increases the affinity of $\operatorname{Trp}$ repressor for operator DNA, leading to the formation of the ternary repressor DNA complex (Rose et al. 1973). The binding of Trp repressor to its operators inhibits the initiation of transcription of the $\operatorname{trpR}$, aroH, and trp operon messages by preventing RNA polymerase from binding to the promoters (Oppenheim et al. 1980).

The physical location of the trp operator was previously defined by deletion studies (Bennett et al. 1976) and by the isolation of constitutive mutations within the trp operator (Bennett and Yanofsky 1978). These operator constitutive mutations map at positions -16 , $-15,-7$, and -6 in the trp promoter sequence. The DNA sequences of the $\operatorname{trp} R$ and $a r o H$ operons reveal sites that exhibit significant sequence homology to the trp operator (Gunsalus and Yanofsky 1980; Singelton et al. 1980; Zurawski et al. 1981). In Figure 1, the sequences of the three operators are aligned to show max-

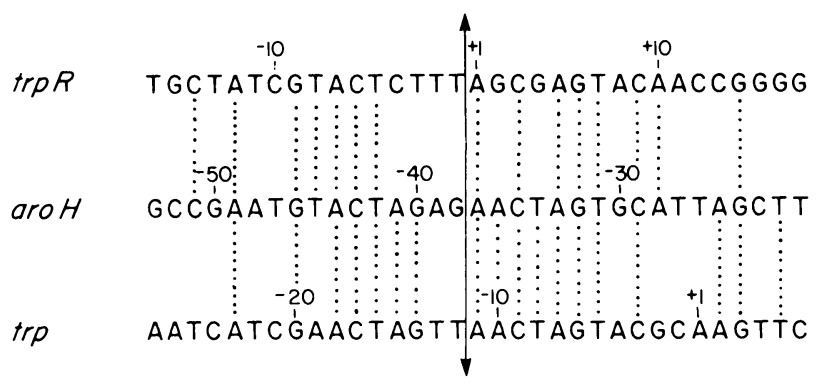

Figure 1. DNA sequence comparison of the operator regions of the $\operatorname{trp} R$, aroH, and $\operatorname{trp}$ operons. The nucleotide sequence of each operator region is numbered relative to the start of transcription. Sequence similarity between the operators is indicated by the vertical dashes. The solid vertical double-headed arrow represents the sequence axis of symmetry. The comparison of the $\operatorname{trp} R$ and $a r o H$ operators shows conservation of 13 of 22 base pairs, the $\operatorname{aroH}$ and $\operatorname{trp}$ sequences have 15 of 22 conserved base pairs, while the $\operatorname{trp} R$ and the $\operatorname{trp}$ operator regions show 16 out of 22 conserved base pairs. No conservation of DNA sequence is seen in the flanking regions of the three operators. 
imum identity. The binding of $\operatorname{Trp}$ repressor at the $\operatorname{tr} p R$ and $a r o H$ operators was demonstrated by a $R s a$ I endonuclease protection assay (Gunsalus and Yanofsky 1980; Zurawski et al. 1981). In this assay bound Trp repressor protects a RsaI endonuclease site, located in the operator, from cleavage by $R$ saI.

In this report, we explore the specific interactions between the $E$. coli Trp repressor and the $\operatorname{trp} R$, aroH, and trp operators by biochemical protection experiments. These interactions are defined by the ability of Trp repressor to protect each operator from methylation by the alkylating agent dimethyl sulfate, or cleavage by the endonuclease DNase I. Comparison of the Trp repressor interactions shows that Trp repressor interacts differently at each operator. The simplest hypothesis that accounts for our data is that Trp repressor binds multiple, helically stacked sites.

\section{Results}

DNase I footprinting of Trp repressor at the trpR, aroH, and trp operators shows differing domains of protection

We have determined the DNase I footprints of Trp repressor at the $\operatorname{trp} R, \operatorname{aroH}$, and $\operatorname{trp}$ operators. DNase I-dependent cleavage reactions were performed in the pres- ence and absence of Trp repressor over a range of different DNA and protein concentrations. DNase I footprints of the $\operatorname{trp} R$, aroH, and trp operators are shown in Figure 2.

The DNase I cleavage pattern for the $\operatorname{trp} R$ operator in the presence of Trp repressor reveals interactions that extend from positions -10 to +15 on the top strand of the $\operatorname{trp} R$ operator and from positions -14 to +12 on the bottom strand. These results are summarized in Figure 3 , and show that the site protected by Trp repressor extends over a 30-bp region, is centered at the start point of transcription, and overlaps the -10 region of the $\operatorname{trp} R$ promoter.

The DNase I pattern for the Trp repressor interactions at the aroH operator is shown in Figure 2B. Protections extend from positions -46 to -22 on the top strand and from positions -54 to -21 on the bottom strand. Trp repressor protects a 33 -bp region that overlaps the -35 region of the aroH promoter.

Figure 2C depicts the changes in the DNase I cleavage pattern of the trp operator in the presence of Trp repressor. These interactions extend from positions -23 to +8 on the top strand and from positions -27 to +8 on the bottom strand. Interactions between the Trp repressor and the trp operator span $35 \mathrm{bp}$, and overlap the -10 region of the trp promoter. This region has also

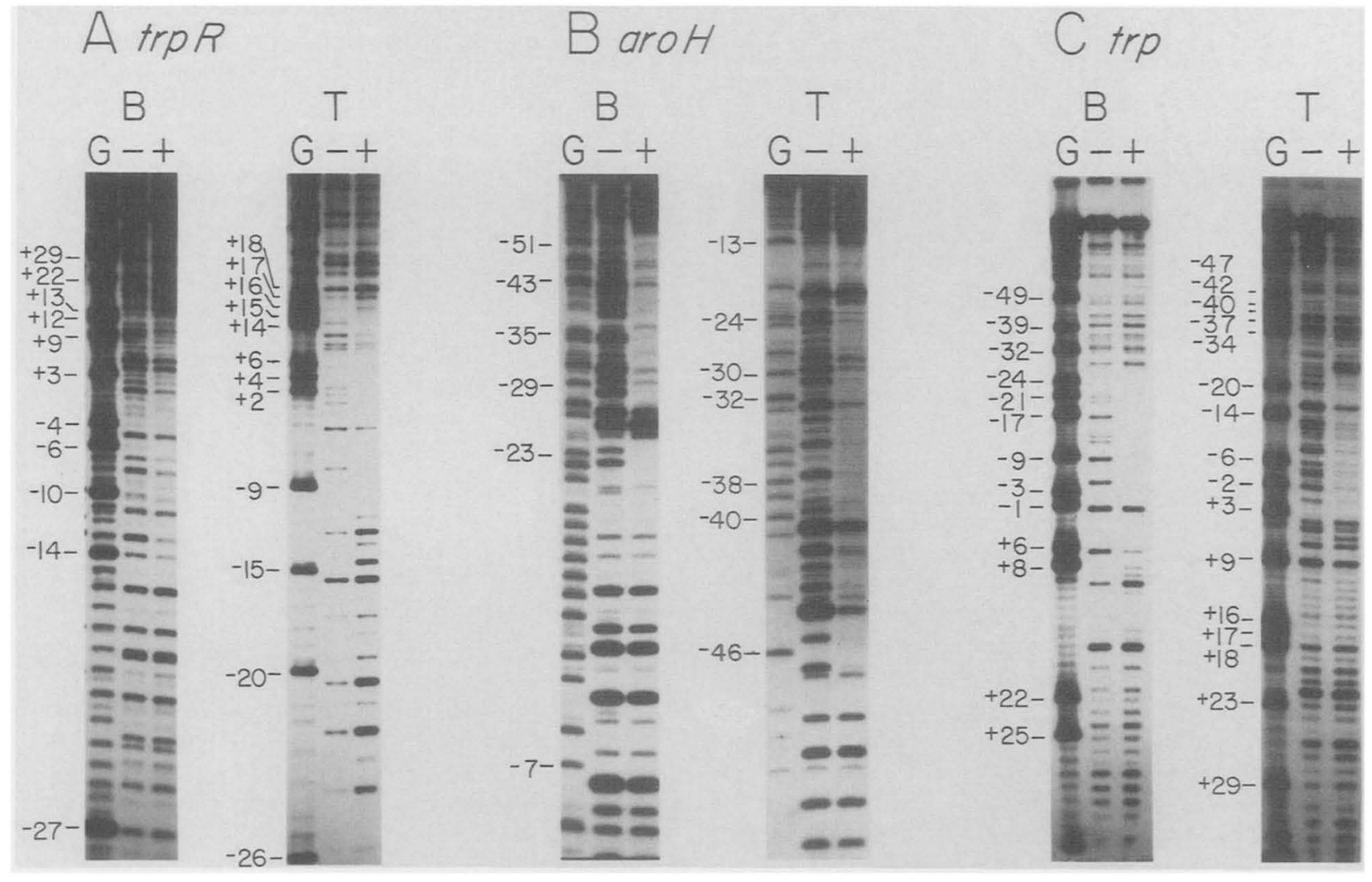

Figure 2. DNase I footprint of Trp repressor binding at the $\operatorname{trp} R$, aroH, and $\operatorname{trp}$ operators. $(A, B$, and $C)$ DNase I footprints of the $\operatorname{trp} R$, aroH, and $\operatorname{trp}$ operators, respectively. In each panel, the $G$ lanes show the DNA guanine cleavage sequence reaction for the bottom (B) and top $(\mathrm{T})$ strands $(\mathrm{A}>\mathrm{G}$ reaction for $a r O H)$ and are used as a reference to align the DNA sequence for the DNase I reaction lanes. The DNase I footprint ladder for each strand in the presence $(+\mid$ and absence $(-)$ of Trp repressor is indicated. Numbering of the DNA sequence is relative to the start of transcription. 
Figure 3. Summary of the DNase I footprint and methylation protection results for Trp repressor binding at the $\operatorname{trp} R$, aroH, and $\operatorname{trp}$ operators. The vertical double-headed arrow represents the DNA sequence axis of symmetry as shown in Fig. 1. The brackets above and below the DNA sequences indicate the DNase I-protected regions (Fig. 2) of each operator for each strand. Guanine residues represented in boldface indicate bases that show decreased methylation by dimethylsulfate in the presence of Trp repressor relative to operator DNA alone.

been identified genetically by constitutive mutations (Bennett and Yanofsky 1978).

The DNase I protection data for the $\operatorname{trpR}$, aroH, and trp operators are compared in Figure 3. Trp repressor protects a different number of base pairs at each operator from DNase I cleavage. The protected region varies from $30 \mathrm{bp}$ for the $\operatorname{tr} p R$ operator to $35 \mathrm{bp}$ for the $\operatorname{tr} p$ operator, and the number and location of repressor-induced hypersensitive DNase I cleavage sites in each operator differs. The protected domains for the $a r o H$ and $\operatorname{trp}$ operators extend beyond the homologous region of the operators (Fig. 1).

\section{Methylation of each operator by dimethyl sulfate}

The results of the DNase I protection experiments show that the region of each operator protected by Trp repressor differs in length. To determine whether this result is due to an idiosyncrasy of the particular DNAmodifying agent we used, we examined the interaction of the Trp repressor with each operator using the chemical methylating agent dimethyl sulfate. In this method, the ability of bound Trp repressor to alter the reactivity of purine imino groups with dimethyl sulfate is revealed by changes in the methylation-dependent cleavage at particular guanine or adenine residues. Data obtained from these studies are shown in Figure 4.

The binding of $\operatorname{Trp}$ repressor at the $\operatorname{trp} R$ operator causes reduced methylation in the top strand for guanines at positions $-9,+2,+4$, and +6 . In the bottom strand, guanines at $-6,-4$, and +9 show reduced methylation. Increased methylation is not detected at residues in either strand. These interactions extend from -9 to +9 , and span 18 bp (Fig. 3). No changes were seen in the cleavage pattern of the adenines, indicating that Trp repressor does not make contacts with the 3 -imino groups of adenines in the minor groove (data not shown).

The effect of Trp repressor binding on the methylation of the aroH operator is shown in Figure 4B. For the top strand, reduced methylation is seen for guanine residues at $-46,-32$, and -24 . The guanine at position -24 is weakly protected from methylation. Increased methylation is seen for guanines at positions $-30,-38$, and -40 . In the bottom strand, reduced methylation is seen

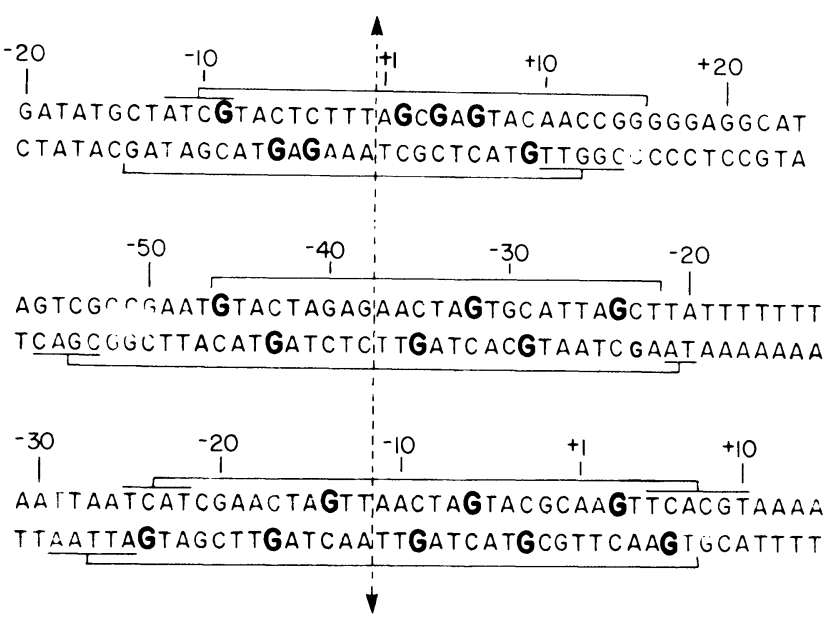

at guanines at $-43,-35$, and -29 . No increased methylation of any guanine residue is observed within the bottom strand of the operator region, and no changes in the methylation pattern of adenines are observed for either strand of the aroH operator (data not shown). The methylation data for the aroH operator are summarized in Figure 3. Methylation protections extend from positions -46 to -24 and span $23 \mathrm{bp}$.

The methylation protection pattern of the trp operator is shown in Figure 4C. For the top strand, changes occur at guanines at positions $-14,-6$, and +3 . The guanine at position +3 shows weak protection. In the bottom strand, changes occur at guanines at positions -24 , $-17,-9,-3$, and +6 . The guanines at positions -24 and +6 show weak protection. No changes in the methylation at adenine residues were seen for either strand (data not shown). The results for both strands of the trp operator are summarized in Figure 3. Methylation protections extend from positions -24 to +6 and span 30 bp.

Comparison of the methylation data for the three operators reveals that Trp repressor interacts differently at each operator (Fig. 3). The size of the operators defined by this method varies from $18 \mathrm{bp}$ for $\operatorname{trp} R$, to $23 \mathrm{bp}$ for aro $H$, and $30 \mathrm{bp}$ for $\operatorname{trp}$, differing in increments of 5-7 bp. The sizes of the $\operatorname{trp} R$, aroH, and $\operatorname{trp}$ operators defined by methylation are smaller than the sizes defined by DNase I footprinting. However, the operators are uniquely ranked in size by both methods. The results obtained by both methods suggest that the Trp repressor makes different, specific contacts in each operator.

The trp operator is comprised of multiple binding sites

One hypothesis that accounts for these results is that Trp repressor binds multiple sites in the aroH and $\operatorname{trp}$ operators. To test this hypothesis, we constructed a plasmid that carries a deletion of the left portion of the trp operator (Fig. 5). We refer to the operator sequence on this plasmid as the mutant trp operator. To examine the binding of Trp repressor at this site, we performed DNase I and methylation protection studies. As shown in Figure 6, the mutant operator is protected from methylation and from DNase I cleavage in the presence of Trp repressor. The results of these studies are summarized in 


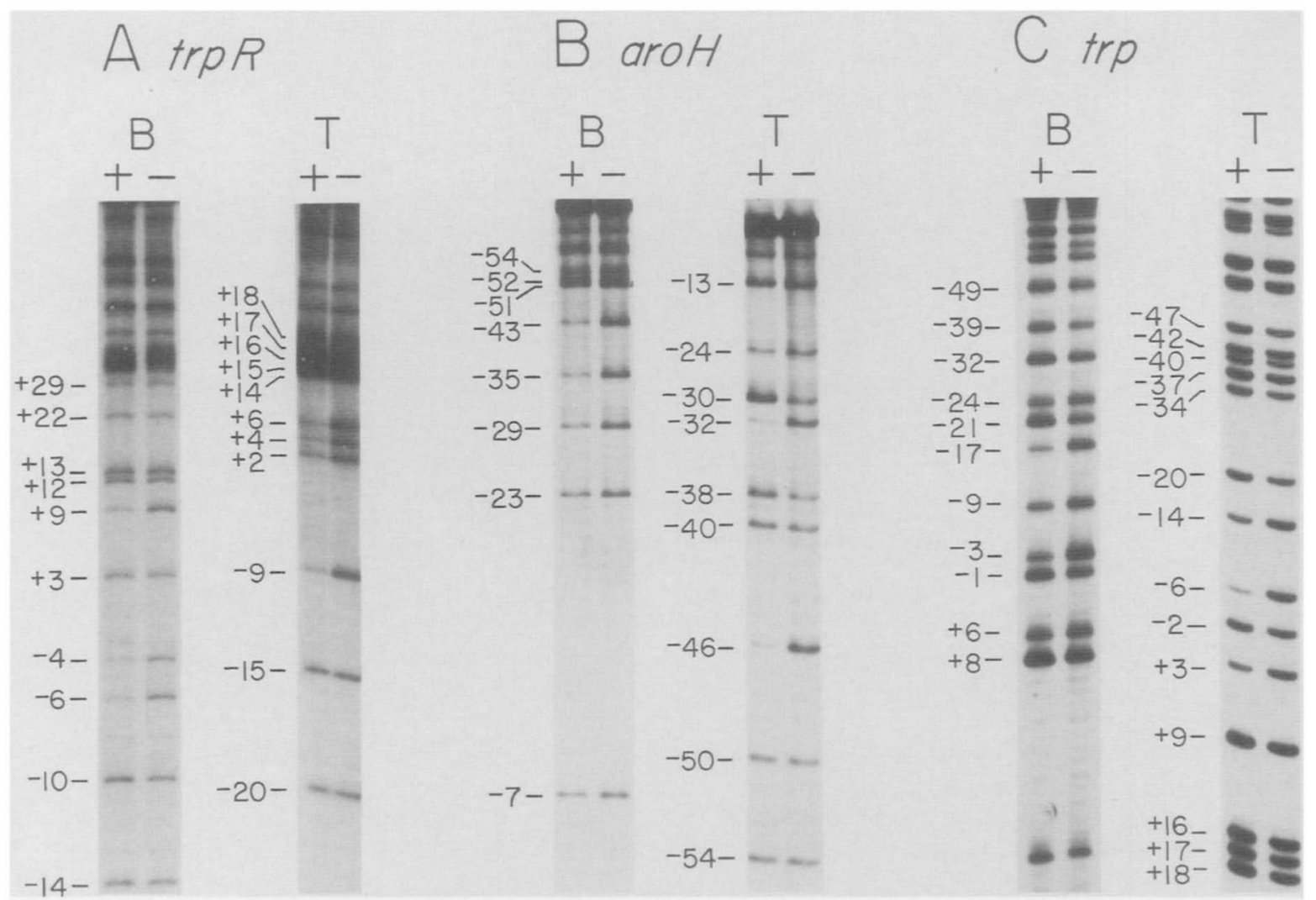

Figure 4. Methylation protection patterns of the $\operatorname{trp} R, \operatorname{aroH}$, and $\operatorname{trp}$ operators. DNA methylation of the three operators by dimethylsulfate in the presence and absence of Trp repressor are shown in $A(\operatorname{trp} R, B(\operatorname{aro} H)$, and $C(\operatorname{trp})$. DNA methylation patterns for the top $(T)$ strand and the bottom (B) strand are indicated for each operator in the presence $(+)$ or absence $(-)$ of Trp repressor. Numbering of the gel ladder is relative to the start of transcription.

Figure 5. Contacts in the homologous portions of the mutant and wild-type trp operators show identical protection patterns with either assay. However, no protection of bases from methylation is observed for the mutant operator in the nonhomologous region substituted for the base pairs to the left of the axis of symmetry. Moreover, the extent of DNase I protection in this substituted region is reduced by $10 \mathrm{bp}$. These results show that at least two Trp repressor binding sites comprise the trp operator, one of which is entirely conserved in the mutant operator, and one of which is at least partially missing in the mutant operator (Fig. 5). In addi- tion, the relative degree to which guanine residues are protected by saturating Trp repressor at homologous positions in the mutant trp and wild-type trp operators is nearly the same (data not shown), which indicates that the maximal occupancy of Trp repressor for the respective binding sites is approximately equal.

\section{Discussion}

Our results show that Trp repressor interacts differently with the $\operatorname{trpR}, a r o H$, and $\operatorname{trp}$ operators. There are differences in the extent of Trp repressor interactions, as de-

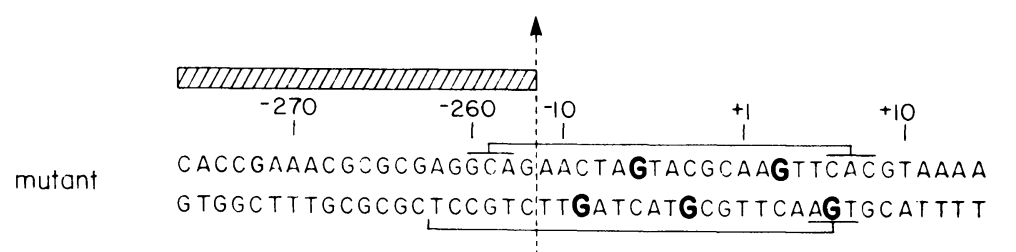

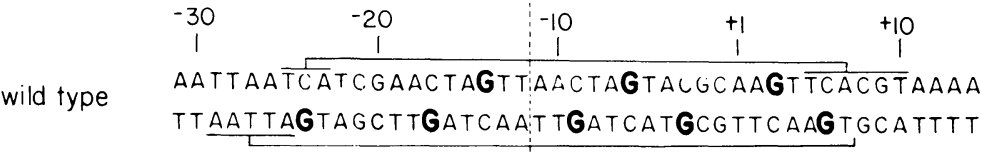

Figure 5. Summary of the DNase I and methylation protection results for the mutant trp operator. The vertical double-headed arrow represents the axis of DNA symmetry of the trp operator as drawn in Fig. 1. The region of the wild-type trp operator deleted in the mutant trp operator is indicated by the bar above the sequence. The brackets above and below the DNA sequences indicate the regions of DNase I protection. The methylation protection results for Trp repressor binding at the half-operator are indicated for bases that show decreased methylation (bold). The trp operator methylation and DNase I data are from Figs. 2 and 4. 


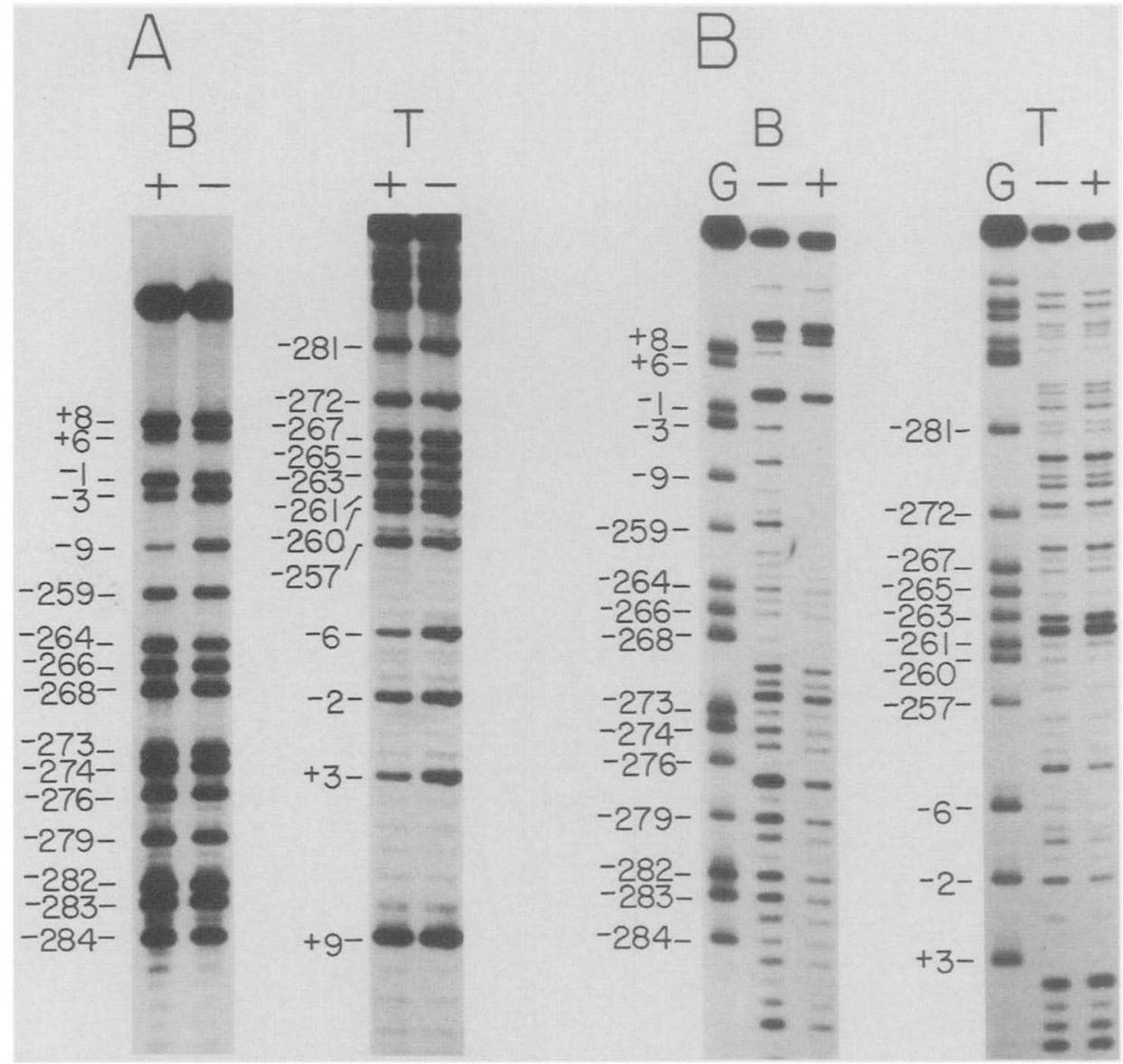

Figure 6. DNase I footprinting and DMS methylation protection of the mutant trp operator by Trp repressor. DMS methylation and DNase I reactions are shown in $A$ and $B$, respectively. Reactions were performed as described in Figs. 2 and 4 and in Materials and methods. The bottom (B) and top (T) strands are indicated. The numbering of the DNA sequence ladder is relative to the start of trp operon transcription.

fined by both assays (Fig. 3). For the trpR operator, $18 \mathrm{bp}$ are protected from methylation and $30 \mathrm{bp}$ are protected from DNase I digestion. For the $a r o H$ operator, $23 \mathrm{bp}$ are protected from methylation and $33 \mathrm{bp}$ are protected from DNase I digestion. For the trp operator, $30 \mathrm{bp}$ are protected from methylation and $35 \mathrm{bp}$ are protected from DNase I digestion. The simplest hypothesis to account for these differences is that the $a r o H$ and trp operators are composed of multiple, helically stacked Trp repressor binding sites.

Figure 3 illustrates that the simplest operator bound by $\operatorname{Trp}$ repressor is the $\operatorname{trpR}$ operator. We assume that the $\operatorname{trpR}$ site is bound by a single repressor dimer and is composed of two operator half-sites. Six of seven guanines protected in this operator are symmetrically paired about its dyad axis and are in two consecutive major grooves on one face of the helix. A nearly symmetric pattern of protected guanines in two consecutive major grooves is seen for the binding of other dimeric repressor proteins, including $\lambda$ Cro protein and $\lambda \mathrm{cI}$ repressor (Johnson et al. 1978). Bass et al. (1987) show that the sequences critical for Trp repressor binding to a symmetric idealized operator are two 5'-CTAG-3' half-site sequences, separated by a 4-bp spacer region. The $\operatorname{trp} R$ operator has three changes in the $5^{\prime}$-CTAG-3' half-sites from the idealized sequence, but none of these changes causes a severe loss of binding affinity in vivo (see Bass et al. 1987). The observed pattern of methylation protection for the $\operatorname{trp} R$ operator is consistent with this minimal definition of a repressor binding site. Two of the three symmetric pairs of protected guanines fall in the 4-bp half-sites. These guanines are in the bottom strand of the left half-site and in the top strand of the right halfsite (Fig. 7). The other symmetric pair of guanines protected from methylation is located 9 bp from the symmetry axis on the opposite strand of each half-site. This position is not critical for Trp repressor binding, since all three symmetric pairs of base pair substitutions at this position have little effect on binding (Bass et al. 1987). It is likely that a nonspecific interaction is responsible for the observed protection.

The interactions of the Trp repressor with the aroH and $\operatorname{trp}$ operators are more complex than with the $\operatorname{trpR}$ operator. Unlike the $\operatorname{trp} R$ operator, which has protected guanines in two consecutive major grooves, the $\mathrm{aroH}$ operator has protected guanines in three consecutive major 
grooves, and the trp operator has protected guanines in four consecutive major grooves.

The protected guanines in the $a r o H$ and $\operatorname{trp}$ operators can be dissected into sets of protection patterns that resemble the protection pattern of the $\operatorname{trp} R$ operator (Fig. 7). We propose that the aroH operator has two helically stacked Trp repressor binding sites and the trp operator has three helically stacked Trp repressor binding sites. The pattern of protection for the mutant trp operator is consistent with the idea that the $\operatorname{trp}$ operator has three sites. The deletion/substitution mutations destroy two of the four half-site sequences in the trp operator. The remaining two half-site sequences constitute a single binding site for a Trp repressor dimer. We reasoned that the pattern of protection observed for the mutant trp operator should resemble the $\operatorname{trp} R$ operator, the simplest natural operator, since both of these operators are composed of a single dimer binding site. The protection data summarized in Figure 5 show that the protected guanines in the mutant trp operator span $15 \mathrm{bp}$, and occur at several positions homologous to the positions of protected guanines in the $\operatorname{trp} R$ operator. Trp repressor makes no contacts to the left of the remaining wild-type sequences in the mutant trp operator. Therefore, no sequences recognized by Trp repressor have been fortuitously introduced to the left of the predicted binding site.

Experiments that measure the relative apparent equilibrium binding constants of the mutant and wild-type trp operators show that the mutant operator binds Trp repressor with an affinity that is only two- to fivefold less than wild type (Haydock et al. 1983; Kumamoto and Gunsalus, unpubl.). This result precludes the possibility that only one Trp repressor dimer makes all of the contacts in the wild-type trp operator. These data further suggest that binding to the helically stacked sites may be cooperative.

Bass et al. (1987) propose that the Trp repressor makes specific contacts with an operator half-site along only one strand of the DNA. Such a model would permit two repressor dimers to contact the same half-site, because the dimers would be positioned roughly $+270^{\circ}$ around the DNA from one another. Alternatively, because the 4 bp in each half-site are a palindrome, both repressor dimers may make identical contacts with each strand of a shared half-site, as do symmetrically related monomers of an endonuclease EcoRI dimer with an EcoRI recognition site (McClarin et al. 1986).

All five of the operator constitutive mutations isolated in the trp operator fall within half-sites shared by two adjacent Trp repressor binding sites (Bennett and Yanofsky 1978). The effects these mutations have on each of the three binding sites correlate with their phenotypes. An $\mathrm{A}: \mathrm{T} \rightarrow \mathrm{T}: \mathrm{A}$ transversion at position -7 causes the greatest loss in repression of the four mutations. This change is predicted to introduce severe defects in the central and rightmost repressor binding sites. The $A: T \rightarrow C: G$, the $G: C \rightarrow T: A$, and the $\mathrm{T}: \mathrm{A} \rightarrow \mathrm{G}: \mathrm{C}$ transversions at positions $-7,-6$, and -16 , respectively, introduce severe defects in the central binding site, and mild defects in the rightmost $1-7$, $-6)$ and leftmost sites $(-16)$, respectively. These mutants retain an intermediate level of repression. Finally, the $\mathrm{A}: \mathrm{T} \rightarrow \mathrm{G}: \mathrm{C}$ transition at position -15 introduces a severe defect into the central binding site and has little effect on the leftmost site (Fig. 7). This mutant operator retains the greatest repression of the four constitutive mutants. It is interesting to note that these constitutive mutations are found only in the shared half-sites of the trp operator, where they may affect two sites simultaneously. This coincidence may explain why Bennett and Yanofsky (1978) failed to isolate constitutive mutations in the outermost half-sites of the trp operator.

The crystal structure and negative dominant mutations of the Trp repressor show that the helix-turn-helix motif is used to recognize a binding site (Kelley and Yanofsky 1985; Schevitz et al. 1985). From computer-assisted docking studies of the Trp repressor with a synthetic 20-bp canonical operator sequence, it is evident that the two helix-turn-helix structures may be positioned so that contacts can be made in two successive major grooves. The location of the helically stacked sites should allow Trp repressor to make classical contacts in all three operators (i.e., each Trp repressor dimer makes

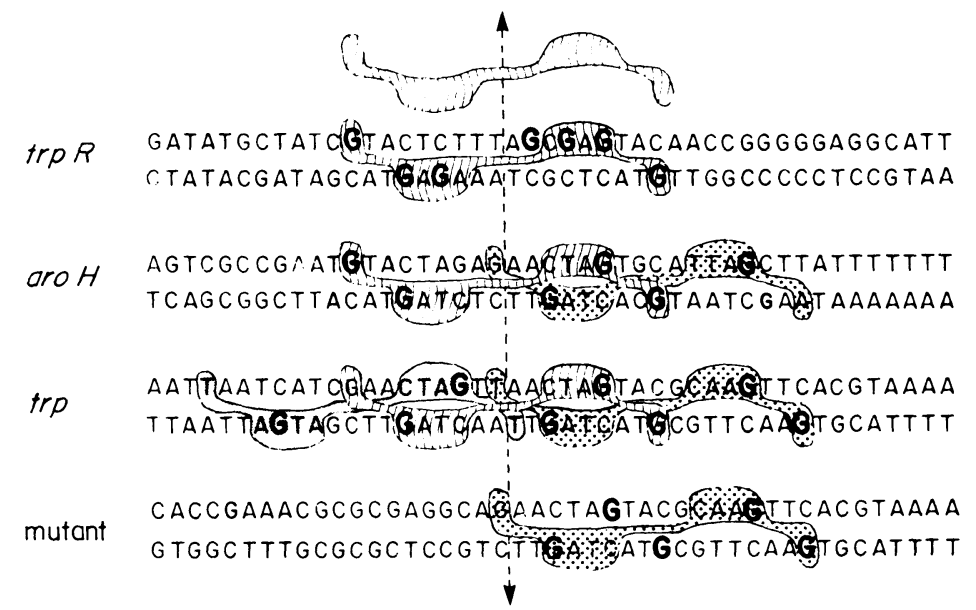

Figure 7. Proposed model for the Trp repressor binding sites at the $\operatorname{trpR}$, aroH, trp, and mutant trp operators. The vertical double-headed arrow represents the DNA sequence axis of symmetry as shown in Fig. 1. Enclosed regions (shaded, hatched, dotted) represent bases that comprise the multiple Trp repressor binding sites at each operator. The $\operatorname{trpR}$, $\operatorname{aroH}, \operatorname{trp}$, and mutant $\operatorname{trp}$ operators contain one, two, three, and one sites, respectively (see text; Bass et al. 1987). Guanine residues represented in boldface indicate bases that show decreased methylation by dimethylsulfate in the presence of Trp repressor relative to operator DNA alone (Figs. 3 and 5). When viewed in three dimensions, the Trp repressor binding sites are tandemly stacked, and together process up the surface of the DNA in helical fashion. 
contacts in two successive major grooves). In contrast, any model in which only one Trp repressor dimer is responsible for all the contacts in each operator must invoke nonclassical interactions in the $a r o H$ and trp operators. The precise location and orientation of Trp repressor on the surface of the operator DNA, however, cannot be resolved until the structure of the proteinDNA co-crystal has been determined.

The $\operatorname{trp} R$, aroH, and $\operatorname{trp}$ operons show differing extents of regulation by $\operatorname{Trp}$ repressor in vivo. The trp operon is regulated by over a 70 -fold range, and the aroH and the $\operatorname{trpR}$ operons are regulated over a four- to fivefold range (Kelley and Yanofsky 1982; Grove and Gunsalus 1987). The role of the multiple stacked Trp repressor binding sites in this differential regulation is unknown. Biochemical and genetic studies are in progress to establish the contribution of these structures to trp regulon control.

\section{Materials and methods}

Bacterial strains and plasmids

E. coli LE392 [hsdR514 ( $\left.\mathrm{r}^{-}, \mathrm{m}^{-}\right)$, supE44, supF58, lacY1, galT22, met $\mathrm{B} 1, \mathrm{trpR} 55]$ was used for plasmid construction and isolation of DNA. Plasmid pRPG5 (trp $\left.\mathrm{R}^{+}\right)$was the source of all trpR operator DNA fragments (Gunsalus and Yanofsky 1980). Plasmid pDNAl containing the $E$. coli aroH operator was the gift of Dennis N. Arvidson (Grove and Gunsalus 1987). The source of trp operator DNA fragments was pKRS101 (Spindler et al. 1984), pRPG78, or pRPG79.

Plasmid pRPG78 contains the trp promoter/operator region and was derived from pKRS101. It was constructed by isolating a 303-bp PvuII-RsaI fragment from pKRS101, which was filled in and inserted into the SmaI site of pRS415 (Simons et al. 1987). The trp promoter/operator fragment contained on a 309 bp EcoRI-BamHI fragment was excised from pRPG78 and combined with the large EcoRI-BamHI fragment of pBR327 to give plasmid pRPG79. The mutant trp operator plasmid pRPG71 was constructed by deleting the 247-bp HpaI fragment of pKRS101. The DNA sequence of the mutant trp operator is shown in Figure 5.

\section{Preparation of labeled DNA fragments}

Preparation and labeling of DNA fragments were performed as described previously (Maniatis 1982). End-labeling at the 5' position was done using T4 polynucleotide kinase and $\left[\gamma^{-32} \mathrm{P}\right] \mathrm{ATP}$ $(3000 \mathrm{Ci} / \mathrm{mmole})$ or by end-filling with an appropriate $\alpha^{-32} \mathrm{P}-\mathrm{la}$ beled deoxynucleotide. Secondary cleavage reactions were performed to give unique 5 '-end-labeled operator containing DNA fragments for the DNase I and methylation protection studies.

For the aroH sense strand studies, the HpaII 249-bp fragment was isolated from pDNAl, 5'-end-labeled, and an HaeIII secondary cleavage reaction performed to yield a singly end-labeled 67-bp aro $H$ operator fragment. For the antisense strand studies, pDNAl was cut with HaeIII, a 351-bp fragment was isolated and 5'-end-labeled, and then cut with HpaII to yield a 5' singly end-labeled 67-bp operator fragment.

For $\operatorname{trp} R$ sense strand studies, pRPG5 was digested with HaeIII, and the 448-bp fragment isolated and 5 '-end-labeled. Secondary cutting with Sau3A yielded a 5 ' singly end-labeled 95-bp fragments. For the $\operatorname{trp} R$ antisense strand, the 187-bp Sau3A fragment was isolated, 5' end-labled, and a secondary cutting reaction performed with Sall to give a 158-bp 5' singly end-labeled fragment.

For DNase I and methylation studies with the trp sense strand, pRPG79 was digested with EcoRI and BamHI, and the 308-bp fragment isolated. Following $5^{\prime}$ end labeling, a secondary cleavage reaction with $H$ haI was performed to give a unique $5^{\prime}$-end-labeled 120-bp trp operator fragment. For studies with the antisense strand, pRPG79 was digested with BamHI. The linearized plasmid was 3 '-end-labeled and then cut with EcoRI to generate a $3^{\prime}$ singly end-labeled 308-bp fragment.

DNA fragments containing the mutant trp operator were prepared from plasmid pRPG71 by BamHI digestion. The ends were labeled, cut with EcoRI, and the 86-bp mutant trp operator fragment isolated.

\section{Trp aporepressor purification}

Trp aporepressor was purified from $E$. coli cells as previously described (Gunsalus and Yanofsky 1980; Arvidson et al. 1987). Analysis of purified Trp aporepressor protein by SDS-polyacrylamide gel electrophoresis revealed the presence of a single protein band $\left(M_{\mathrm{r}}=12,500\right)$. The purity was estimated to be greater than $99 \%$. The Trp aporepressor preparations were greater than $90 \%$ active for operator DNA binding as determined by a DNase I protection gel assay (Kumamoto and Gunsalus, unpubl.). Protein concentration was determined by absorbance at $280 \mathrm{~nm}\left(\mathrm{E}_{1 \%}=1.2 \mathrm{~cm}^{-1} \mathrm{mg}^{-1}\right.$; Joachimiak et al. 1983).

\section{DNase footprinting of operator DNA}

For standard DNase I footprinting experiments, approximately 20 pmoles of purified Trp aporepressor was added to approximately 1 pmole of ${ }^{32} \mathrm{P}$-labeled operator DNA fragment in $1 \mu \mathrm{l}$ of DNase I reaction buffer $(30 \mathrm{~mm}$ Tris- $\mathrm{HCl}, \mathrm{pH} 8,100 \mathrm{~mm} \mathrm{KCl}, 3$ $\mathrm{mM} \mathrm{MgCl}, 2 \mathrm{~mm}$ DTT, 2 mM EDTA, $2 \mu \mathrm{g}$ sonicated salmon sperm DNA, $\pm 1 \mathrm{mM}$ L-tryptophan; Galas and Schmitz 1978). The final concentrations of aporepressor and operator used in these experiments were typically in the range of 500 and $5 \mathrm{nM}$, respectively, and are at physiological levels (Gunsalus et al. 1986). All of the DNase I and DMS methylation experiments were performed in the presence of salmon sperm DNA (nonspecific carrier DNA). The operator DNA was present at $20 \mathrm{ng}$ versus $2000 \mathrm{ng}$ of the carrier DNA, which corresponds to a 100 fold excess on a weight/weight basis, or a 1000-fold molar excess of nonspecific DNA binding sites relative to operator sites. DNase I was diluted in DNase I buffer supplemented with 2 $\mathrm{mM} \mathrm{CaCl}{ }_{2}$ and added at a final concentration of $10 \mu \mathrm{g} / \mathrm{ml}$ to initiate the reaction. The mixture was incubated at $37^{\circ} \mathrm{C}$ for 10 min and the reaction was then quenched by the addition of 75 $\mu l$ of DNase I stop solution (Galas and Schmidt 1978). The DNA mixture was phenol-treated and desalted on a Sephadex G-25 column, and the DNA fragments were precipitated by addition of two volumes of ethanol. Sequence analysis of labeled DNA fragments (Maxam and Gilbert 1977) and DNA polyacrylamide gel electrophoresis (Sanger and Coulson 1978) were performed as described.

\section{Chemical methylation of operator DNA by dimethyl sulfate}

Methylation protection reactions with each strand of the trp, $\operatorname{trp} R$, and $a r o H$ operators were carried out using a standard reaction protocol. Operator DNA $/ \sim 1$ pmole of 5 ' end-labeled fragment) was added to $200 \mu \mathrm{l}$ of dimethyl sulfate reaction buffer (Gilbert et al. 1976) containing $2 \mu \mathrm{g}$ of sonicated salmon sperm DNA. Where indicated, L-tryptophan was present at a final concentration of $1 \mathrm{mM}$ and Trp repressor was added to the reac- 
tion mixture at a final concentration of $250 \mathrm{nM}$. The mixture was preincubated on ice for $10 \mathrm{~min}$ and the reaction was initiated by addition of dimethyl sulfate $(1 \mu \mathrm{l})$. After $5 \mathrm{~min}$ at $20^{\circ} \mathrm{C}$, the methylation reaction was stopped by the addition of DMS stop solution $(50 \mu \mathrm{l})$, the DNA fragments were precipitated $(2 \times)$, washed once with $75 \%$ ethanol, and dried (Maxam and Gilbert 1977). DNA samples were then treated according to the alternative guanine cleavage ( $\mathrm{G}$ reaction) or the strong adenine/ weak guanine cleavage ( $A>G$ reaction) procedures of Maxam and Gilbert (1977). DNA sequence analysis and gel electrophoresis were performed as described (Maxam and Gilbert 1977; Sanger and Coulson 1978).

\section{Enzymes and reagents}

Calf intestinal alkaline phosphatase was obtained from Boehringer Mannheim (Indianapolis, Indiana), restriction enzymes (HaeIII, HpaII, BamHI, and EcoRI) were obtained from BRL (Gaithersburg, Maryland) (Sau3A and SalI) or from New England Biolabs (Beverly, Massachusetts), and T4 polynucleotide kinase was purchased from Pharmacia-PL Biochemicals (Piscataway, New Jersey). [ $\gamma^{-32}$ P]ATP $(3000 \mathrm{Ci} / \mathrm{mmole})$ and $\left[\alpha-{ }^{32} \mathrm{P}\right] \mathrm{dATP}(3000 \mathrm{Ci} / \mathrm{mmole})$ were obtained from Amersham (Arlington Heights, Illinois). Dimethyl sulfate was purchased from Aldrich Chemical Corporation (Milwaukee, Wisconsin).

\section{Acknowledgments}

We thank Philip Youderian for critical comments on the manuscript. This work was supported by a grant from the National Institutes of Health (GM 29456) and by a UCLA Biochemical Research Grant to R.P.G. A.A.K. was supported in part by a Public Health Service Genetics Training Grant (GM07104) from the National Institutes of Health.

\section{References}

Arvidson, D.N., C. Bruce, and R.P. Gunsalus. 1986. Interaction of the Trp repressor with its ligand, L-tryptophan. I. Biol. Chem. 261: 238-243.

Arvidson, D.N., A.A. Kumamoto, and R.P. Gunsalus. 1987. A simple three-step batch purification of the Escherichia coli Tryptophan repressor. Protein purification: Micro to macro. UCLA Symp. Mol. Cell. Biol. New Series 68: (in press).

Bachman, B.J. 1983. Linkage map of Escherichia coli K-12, 7 ed. Microbiol. Rev. 47: 180-230.

Bass, S., P. Sugiono, D.N. Arvidson, R.P. Gunsalus, and P. Youderian. 1987. DNA specificity determinants of Escherichia coli tryptophan repressor binding. Genes Dev. 1: 000-000.

Bennett, G.N., M.E. Schweingruber, K.D. Brown, C. Squires, and C. Yanofsky. 1976. Nucleotide sequence of region preceding trp mRNA initiation site and its role in promoter and operator function. Proc. Natl. Acad. Sci. 73: 2351-2355.

Bennett, G.N. and C. Yanofsky. 1978. Sequence analysis of operator constitutive mutants of the tryptophan operon of Escherichia coli. J. Mol. Biol. 121: 179-192.

Brown, K.D. 1968. Regulation of aromatic amino acid biosynthesis in Escherichia coli K12. Genetics 60: 31-48.

Cohen, G. and F. Jacob. 1959. Sur la repression de la synthese des enzymes intervenant dans la formation du tryptophane chez Escherichia coli. C.R. Acad. Sci. Ser. D. 248: 34903492.

Galas, D.J. and A. Schmitz. 1978. DNase footprinting: A simple method for the detection of protein-DNA binding specificity. Nucleic Acids Res. 5: 3157-3170.
Gilbert, W., A. Maxam, and A.D. Mirzabekov. 1976. Control of ribosome synthesis, The Alfred Benson Symposium IX led. N.O. Kjelgaard and O. Maaloe), pp. 139-148. Munksgaard, Copenhagen.

Grove, C. and R.P. Gunsalus. 1987. Regulation of the aroH operon of Escherichia coli by the tryptophan repressor. I. Bacteriol. 169: 2158-2164.

Gunsalus, R.P. and C. Yanofsky. 1980. Nucleotide sequence and expression of Escherichia coli trpR, the structural gene for the trp aporepressor. Proc. Natl. Acad. Sci. 77: 71177121.

Gunsalus, R.P., A. Gunsalus-Miguel, and G.L. Gunsalus. 1986. Intracellular Trp repressor levels in Escherichia coli. J. Bacteriol. 167: 272-278.

Haydock, P.N., G. Bogosian, K. Brechling, and R.L. Somerville. 1983. Studies on the interaction of $\operatorname{trp}$ holorepressor with several operators. J. Mol. Biol. 170: 1019-1030.

Joachimiak, A., R.L. Kelley, R.P. Gunsalus, C. Yanofsky, and P.B. Sigler. 1983. Purification and characterization of Trp aporepressor. Proc. Natl. Acad. Sci. 80: 668-672.

Johnson, A., B.J. Meyer, and M. Ptashne. 1978. Mechanism of action of the cro protein of bacteriophage $\lambda$. Proc. Natl. Acad. Sci. 75: 1783-1787.

Kelley, R.L. and C. Yanofsky. 1982. Trp aporepressor production is controlled by autogenous regulation and inefficient translation. Proc. Natl. Acad. Sci. 79: 3120-3124.

1985. Mutational studies with the Trp repressor of Escherichia coli support the helix-turn-helix model of repressor recognition of operator DNA. Proc. Natl. Acad. Sci. 82: $483-487$.

Maniatis, T., E.F. Fritsch, and I. Sambrook. 1982. Molecular cloning: A laboratory manual. Cold Spring Harbor Laboratory, Cold Spring Harbor, New York.

Maxam, A. and W. Gilbert. 1977. A new method for sequencing DNA. Proc. Natl. Acad. Sci. 74: 560-564.

McClarin, J.A., C.A. Frederick, B.C. Wang, P. Greene, H.W. Boyer, J. Grable, and J.M. Rosenberg. 1986. Structure of the DNA-EcoRI endonuclease complex at $3 \mathrm{~A}$ resolution. Science 234: 1526-1541.

Morse, D. and C. Yanofsky. 1969. Amber mutants of the $\operatorname{trp} R$ regulatory gene. I. Mol. Biol. 44: 185-193.

Oppenheim, D.S., G.N. Bennett, and C. Yanofsky. 1980. Escherichia coli RNA polymerase and Trp repressor interaction with the promoter-operator region of the tryptophan operon of Salmonella typhimurium. I. Mol. Biol. 144: 133-142.

Pittard, J., L. Camakaris, and B.J. Wallace. 1969. Inhibition of 3-Deoxy-D-arabinohepulosonic acid-7-phosphate synthetase $($ trp $)$ in Escherichia coli. J. Bacteriol. 97: 1241-1247.

Rose, J.K., C.L. Squires, C. Yanofsky, H. Yang, and G. Zubay. 1973. Tryptophanyl-tRNA and tryptophanyl tRNA synthetase are not required for in vitro repression of the tryptophan operon. Nature New Biol. 245: 133-137.

Sanger, F. and A.R. Coulson. 1978. The use of thin acrylamide gels for DNA sequencing. FEBS Lett. 87: 107-110.

Schevitz, R.W., Z. Otwinowski, A. Joachimiak, C.L. Lawson, and P.B. Sigler. 1985. The three-dimensional structure of $t r p$ repressor. Nature 317: 782-786.

Simons, R.W., F. Houman, and N. Kleckner. 1987. Improved multicopy lac-based protein and operon fusion cloning tools. Gene (in press).

Singleton, C.K., W.D. Roeder, G. Bogosian, R.L. Somerville, and H.L. Weith. 1980. DNA sequence of the E. coli $\operatorname{trp} R$ gene and prediction of the amino acid sequence of $\operatorname{Trp}$ repressor. Nucleic Acid Res. 8: 1551-1560.

Spindler, K.R., D.S. Rosser, and A.J. Berk. 1984. Analysis of adenovirus transforming proteins from early regions $1 \mathrm{~A}$ and 
Kumamoto et al.

1B with antisera to inducible fusion antigens produced in Escherichia coli. J. Virol. 49: 132-141.

Yanofsky, C. 1971. Tryptophan biosynthesis in Escherichia coli. J. Am. Med. Assoc. 218: 1026-1035.

Zurawski, G., R.P. Gunsalus, K.D. Brown, and C. Yanofsky. 1981. Structure and regulation of $a r o H$, the structural gene for the tryptophan-repressible 3-Deoxy-D-arabino-heptulosonic acid-7-phosphate synthetase of Escherichia coli. I. Mol. Biol. 145: 47-73. 


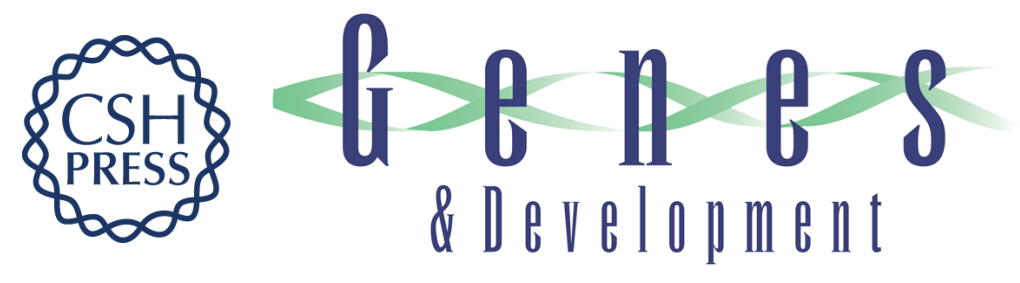

\section{Escherichia coli tryptophan repressor binds multiple sites within the aroH and trp operators.}

A A Kumamoto, W G Miller and R P Gunsalus

Genes Dev. 1987, 1:

Access the most recent version at doi:10.1101/gad.1.6.556

$\begin{array}{ll}\text { References } & \text { This article cites } 28 \text { articles, } 14 \text { of which can be accessed free at: } \\ \text { http://genesdev.cshlp.org/content/1/6/556.full.html\#ref-list-1 }\end{array}$

License

Email Alerting Receive free email alerts when new articles cite this article - sign up in the box at the top Service right corner of the article or click here.

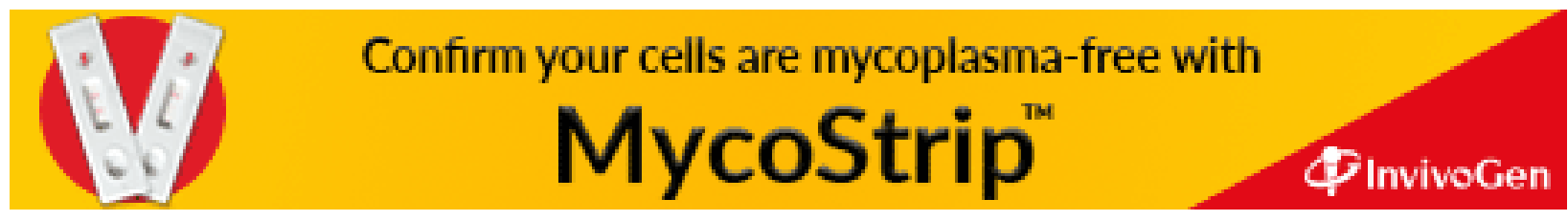

